\title{
Small RNA Bidirectional Crosstalk During the Interaction Between Wheat and Zymoseptoria tritici
}

\author{
Xin $\mathrm{Ma}^{*}$, Jasmin Wiedmer and Javier Palma-Guerrero* \\ Plant Pathology, Institute of Integrative Biology, ETH Zurich, Zurich, Switzerland
}

Cross-kingdom RNA interference (RNAi) has been shown to play important roles during plant-pathogen interactions, and both plants and pathogens can use small RNAs (sRNAs) to silence genes in each other. This bidirectional cross-kingdom RNAi was still unexplored in the wheat-Zymoseptoria tritici pathosystem. Here, we performed a detailed analysis of the sRNA bidirectional crosstalk between wheat and Z. tritici. Using a combination of small RNA sequencing (sRNA-seq) and microRNA sequencing (mRNA-seq), we were able to identify known and novel sRNAs and study their expression and their action on putative

OPEN ACCESS

Edited by:

Brigitte Mauch-Mani,

Université de Neuchâtel, Switzerland

Reviewed by:

Dirk Albert Balmer,

Syngenta, Switzerland

Amir Mirzadi Gohari,

University of Tehran, Iran

${ }^{*}$ Correspondence:

Xin $\mathrm{Ma}$

xin.ma@usys.ethz.ch

Javier Palma-Guerrero

javier.palma@usys.ethz.ch

Specialty section: This article was submitted to Plant Microbe Interactions,

a section of the journal

Frontiers in Plant Science

Received: 08 July 2019 Accepted: 27 November 2019

Published: 08 January 2020

Citation:

Ma $X$, Wiedmer $J$ and

Palma-Guerrero J (2020)

Small RNA Bidirectional Crosstalk During the Interaction Between Wheat and Zymoseptoria tritici.

Front. Plant Sci. 10:1669.

doi: 10.3389/fp/s.2019.01669 targets in both wheat and $Z$. tritici. We predicted the target genes of all the sRNAs in either wheat or $Z$. tritici transcriptome and used degradome analysis to validate the cleavage of these gene transcripts. We could not find any clear evidence of a cross-kingdom RNAi acting by mRNA cleavage in this pathosystem. We also found that the fungal sRNA enrichment was lower in planta than during in vitro growth, probably due to the lower expression of the only Dicer gene of the fungus during plant infection. Our results support the recent finding that $Z$. tritici sRNAs cannot play important roles during wheat infection. However, we also found that the fungal infection induced wheat sRNAs regulating the expression of specific wheat genes, including auxin-related genes, as an immune response. These results indicate a role of sRNAs in the regulation of wheat defenses during Z. tritici infection. Our findings contribute to improve our understanding of the interactions between wheat and $Z$. tritici.

Keywords: Triticum aestivum L, Zymoseptoria tritici, small RNAs, RNAi, sRNA-seq, degradome analysis

\section{INTRODUCTION}

Small RNAs (sRNAs) are short non-coding RNAs (approximately 20-30 nucleotides in length) that play important roles in the regulation of gene expression and genome stability in eukaryotic organisms (Moazed, 2009). The three major classes of sRNAs are microRNAs (miRNAs), small interfering RNAs (siRNAs), and PIWI-associated RNAs (piRNAs) (Carthew and Sontheimer, 2009; Moazed, 2009). While many miRNAs and siRNAs are conserved among plants, piRNAs have so far only been found in animal cells and are necessary in the development of germ cells by silencing transposons or other genetic elements (Vagin et al., 2006; Siomi et al., 2011; Axtell, 2013).

In plants, miRNAs $(\sim 21 \mathrm{nt})$ are produced from imperfect fold-back structures of selfcomplementary non-coding transcripts, originated from endogenous loci, and they regulate 
target mRNAs by posttranscriptional gene silencing (PTGS). In contrast, siRNAs derive from double-strand RNA (dsRNA) with near-perfect complementarity, and they can be both of endogenous origin, e.g., inverted repeat (IR) and dsRNA synthesized by endogenous RNA-dependent RNA polymerases (RDRs) (Allen et al., 2005; Felippes and Weigel, 2009; Fei et al., 2013; Creasey et al., 2014), or exogenous origin, e.g., viral intermolecular replication intermediates or intramolecular secondary structures. siRNAs guide either PTGS (mediated by 21/22-nt siRNAs) or RNA-directed DNA methylation (RdDM) often resulting in transcriptional gene silencing (TGS; mediated by 24-nt siRNAs) (Matzke and Mosher, 2014; Borges and Martienssen, 2015). While each sRNA pathway possesses specific characteristics in terms of biogenesis and accessory proteins, the basic mechanism of RNA silencing shares few consensus biochemical steps: i) initiation by dsRNA, ii) dsRNA processing to generate the mature sRNAs by Dicer (or Dicerlike, DCL) proteins, and iii) sRNA incorporation into Argonaute (AGO) proteins to interact with target mRNA or DNA in order to execute their silencing functions (Liu et al., 2004; Hutvagner and Simard, 2008; Bologna and Voinnet, 2014).

sRNAs are involved in plant development, reproduction, and genome reprogramming and contribute to the phenotypic plasticity of plants (Borges and Martienssen, 2015). Besides, plant sRNAs also mediate the response to abiotic stresses, such as water stress, sulfate stress, phosphate starvation, and cold stress (Sunkar and Zhu, 2004; Sunkar et al., 2007; Park et al., 2010; Guleria et al., 2011; Kamthan et al., 2015). Plants as well employ RNA interference (RNAi) as defense strategies against biotic stress (Ruiz-Ferrer and Voinnet, 2009; Zhang et al., 2011; Weiberg et al., 2014). In Arabidopsis, miR393 is induced during bacterial infection, and it suppresses the auxin signaling by targeting auxin response TIR1 and three related F-box genes to inhibit pathogen growth (Achard et al., 2007; Chen et al., 2007; Navarro et al., 2008; Grant and Jones, 2009). Besides, plant miRNAs can also regulate NBS-LRR transcripts as an important immune response to pathogens. (Zhai et al., 2011; Li et al., 2012; Shivaprasad et al., 2012; Fei et al., 2013).

In fungi, RNAi is an ancient host defense mechanism against viruses and transposons invasion (Chang et al., 2012; Nicolas and Ruiz-Vazquez, 2013; Torres-Martinez and Ruiz-Vazquez, 2017). However, no miRNAs, but only miRNA-like RNAs (milRNAs), have been identified in fungi (Lee et al., 2010; Goodwin et al., 2011), which share similarities with conventional miRNAs, but are synthesized by different mechanisms (Lee et al., 2010). The first RNAi process in fungi, called quelling, was found in Neurospora crassa. Quelling is triggered by repetitive transgenes during mitotic growth, and it is essential for suppressing transposons replication (Romano and Macino, 1992; Nolan et al., 2005). QiRNAs, a class of QuellingDeficient 2 (QDE2) interacting sRNAs, are specifically induced when Neurospora is treated with a DNA-damaging agent (Lee et al., 2009). Additionally, sRNAs are also required for fungal growth and pathogenesis in Magnaporthe oryzae (Raman et al., 2017).
RNAi has recently been reported to play important roles in plant and pathogen interactions. In host-induced gene silencing (HIGS), accumulation of double-stranded or antisense RNAs in barley and wheat can target and silence genes in the fungal pathogen Blumeria graminis as an effective defense strategy (Nowara et al., 2010). This cross-kingdom RNAi has also been shown to happen in a natural way. A fungal pathogen, Botrytis cinerea, can hijack host plant defenses by using small RNAs that bind to the plant AGO1 and silence plant defense-related genes (Weiberg et al., 2013). A novel milRNA has been identified in Puccinia striiformis $\mathrm{f}$. sp. tritici (Pst) that silences a wheat pathogenesis-related 2 gene and influences the plant immunity (Wang et al., 2017). Plants can also use the same RNAi mechanisms to defend against pathogenic fungi, e.g., cotton plants can export their miRNAs into the fungal pathogen Verticillium dahliae and inhibit the expression of virulence genes during the infection cycle (Wang et al., 2016; Zhang et al., 2016). In addition, a recent study has shown that Arabidopsis can send sRNAs in extracellular vesicles to fungal pathogens, revealing how sRNAs travel from hosts to fungal pathogens (Cai et al., 2018).

Zymoseptoria tritici causes septoria tritici blotch, which is an important disease of wheat worldwide and the most damaging disease on wheat in Europe (Hardwick et al., 2001; O'Driscoll et al., 2014). After penetrating wheat leaves through stomata, $Z$. tritici starts the complex infection cycle characterized by a long period of asymptomatic growth known as a latent period that can last up to 2 weeks (Sanchez-Vallet et al., 2015). This latent period is followed by a rapid switch to necrotrophic growth in which a large number of plant cells are killed in a 2- to 3-day period, and it is accompanied by a rapid accumulation of fungal biomass inside the plants (Rudd et al., 2015). Even though there are many predicated candidate effectors in Z. tritici (Mirzadi Gohari et al., 2015; Rudd et al., 2015; Palma-Guerrero et al., 2016), so far, only LysM has been confirmed to be required for virulence, and it can suppress PAMP-triggered immunity during the infection (Marshall et al., 2011; Mirzadi Gohari et al., 2015). Therefore, the mechanisms underlying $Z$. tritici pathogenicity still remain largely unknown. In previous studies, we have analyzed the transcriptomes of both wheat and $Z$. tritici during the infection (Palma-Guerrero et al., 2017; Ma et al., 2018b). These studies revealed the mutual transcriptomic responses between wheat and $Z$. tritici during the interactions, which, together with other transcriptomics studies in this pathogen, shows that a whole genome-wide reprogramming of gene expressions happens in both plant and fungus (Rudd et al., 2015; Palma-Guerrero et al., 2017; Ma et al., 2018b). A recent study reported that the deletion of RNAi-related genes in $Z$. tritici is dispensable for full infection of wheat. Conversely, they also showed that HIGS is not effective to control Z. tritici (Kettles et al., 2019). This work indicates that Z. tritici sRNAs do not play important roles in a cross-kingdom RNAi manner, contrary to previous studies in other fungal plant pathogens (Weiberg et al., 2013; Wang et al., 2017). But how wheat sRNAs take part in this interaction still remains unexplored. The investigation of this natural bidirectional RNAi between wheat and $Z$. tritici at the same time in the 
same samples can provide a detailed scenario of this hidden interactions.

Here, we analyzed the small RNAs produced during wheat infection by $Z$. tritici during the latent period and the transition phase of the disease cycle to identify both the $Z$. tritici sRNAs and the wheat sRNAs and their degraded targets. We found fungal sRNAs induced during infection and predicted their targeted genes in the wheat transcriptome. But cleavage of these predicted targets could not be confirmed by degradome analysis. Additionally, the expression of the fungal Dicer gene was downregulated and barely expressed in planta. Conversely, wheat sRNAs involved in the regulation of auxin signaling were also induced in response to $Z$. tritici. Thus, our results suggest that the cross-kingdom RNAi in wheat and $Z$. tritici pathosystem does not lead to detectable mRNA degradation by PTGS during infection. These findings support the recent published work of fungal sRNAs in wheat and $Z$. tritici pathosystem (Kettles et al., 2019). However, we also show that wheat sRNAs can be used as an immune response to the infection by $Z$. tritici.

\section{MATERIALS AND METHODS}

\section{Samples Collection and RNA Extractions}

The total RNAs from leaf samples of wheat cultivar Drifter infected with the $Z$. tritici $3 \mathrm{D} 7$ strain at 7,12 , and 14 days postinfection (dpi) were obtained in a previous study from our group (Palma-Guerrero et al., 2017). The RNAs from uninfected mock samples at 7,12, and $14 \mathrm{dpi}$ that serve as controls were obtained in this study by using the control samples generated in the same previous study (Palma-Guerrero et al., 2017). The RNA samples of the fungus growing in vitro were generated in a different previous study (Francisco et al., 2018). Three replicates per sample with the highest RNA quality according to Bioanalyzer 2100 (Agilent) were used as biological replicates for all the library preparations and sequencing. All the raw sequencing data were deposited into the NCBI Short Read Archive under the accession number SRP154808.

\section{Small-RNA-Seq Analysis}

The small-RNA-seq libraries were constructed using the TruSeq small-RNA kit and sequenced by HiSeq4000 by the Functional Genomic Center of Zurich (FGCZ). Reads were trimmed to remove sequencing adapters and keep only insert with length $\geq 15$ using Trimmomatic-0.36 (Bolger et al., 2014) with the settings "ILLUMINACLIP:smallRNA-SE.fa:2:30:10 LEADING:2 TRAILING:2 SLIDINGWINDOW:4:15 MINLEN:15". The adapter sequence in smallRNA-SE.fa is “TGGAATTCTCGGGTGCCAAGG”.

\section{Wheat sRNA Predictions}

All the clean reads were collapsed using fastx-ToolKit (Gordon and Hannon, 2010). Wheat transfer RNAs (tRNAs), ribosomal RNAs (rRNAs), and small nucleolar RNAs (snoRNAs) were removed. The sequences of tRNAs, rRNAs, and snoRNAs were extracted from Triticum_aestivum.TGACv1.ncrna.fa on Ensembl plant (Kersey et al., 2018).
We used miRDeep-p (Yang and Li, 2011) and ShortStack (Shahid and Axtell, 2014; Johnson et al., 2016) for the wheat miRNA predictions. For miRDeep-p, all the filtered reads were mapped against wheat genome International Wheat Genome Sequencing Consortium (IWGSC) RefSeq v1.0 (Appels et al., 2018) using bowtie (Langmead et al., 2009), allowing no mismatches. The mapped reads from all the samples including infected samples and mocks were merged together and collapsed. We used the mapped reads to retrieve miRNAs annotated in IWGSC RefSeq v1.0 annotations (Appels et al., 2018). We used miRDeep-P (Yang and Li, 2011) to predict wheat miRNAs, setting the length of candidate precursors as 300 nt. A customized Perl script was used to filter the miRNAs based on the new criteria of plant miRNA annotation (Axtell and Meyers, 2018). Basically, 1) both the miRNA and miRNA* are predicted and expressed in the sequencing data; 2) the expression of miRNA is more than 10 reads per million in at least one of the samples; 3 ) the length of mature miRNAs should be between 20 and $24 \mathrm{nt}$; 4) the mismatches between miRNA and miRNA* are under 5 ; 5) the asymmetric mismatches between miRNA and miRNA $^{*}$ are under 3 ; and 6 ) the length of precursor should be under $300 \mathrm{nt}$.

We as well used ShortStack (Shahid and Axtell, 2014; Johnson et al., 2016) to predict the wheat sRNA loci with the settings "mismatches 0 -mincov $0.5 \mathrm{rpm}$." Three replicates of each condition were merged together for the ShortStack alignment and annotation. Only the sRNA candidate loci with expressions of at least $10 \mathrm{rpm}$ in at least two replicates of each condition were kept. Then the major siRNAs and miRNAs from every condition were summarized together. We named every unique siRNA and miRNA reads as miRNA-uniq or siRNA, and with ascending order numbers. We combined the annotations of miRNAs from Arabidopsis, rice, and wheat from miRBase and the annotations of wheat miRNA from IWGSC together to make a customized database of the known plant miRNAs (Griffiths-Jones, 2004; Griffiths-Jones et al., 2006; Griffiths-Jones et al., 2008; Kozomara and Griffiths-Jones, 2011; Kozomara and Griffiths-Jones, 2014). All the unique mature sequences of predicted miRNAs were used to blast (e-value under 10) against the customized database to detect if the miRNAs were previously annotated (Camacho et al., 2009).

All the sequences of mature sRNAs were used to count sRNA expression in each sample. We used TMM (trimmed mean of $M$ values) (Robinson and Oshlack, 2010) to normalize the library sizes and RPM (reads per million) to describe the sRNA expression. The differentially expressed sRNAs were called by edgeR (Robinson et al., 2010) using $\log$ (fold change) $\geq 1$ (or -1 or less) and FDR $<0.05$.

\section{Fungal sRNA Predictions}

The fungal tRNAs, rRNAs, and snoRNAs were removed from clean reads. The annotations of tRNAs, rRNAs, and snoRNAs were extracted from Ensembl fungi (Goodwin et al., 2011; Kersey et al., 2018). The published genome reference of 3D7 was used in the analysis (Plissonneau et al., 2016). Then the filtered reads were used to predict fungal sRNAs with ShortStack using the same strategy as described above for the plant. The expression of 
the fungal sRNAs were obtained as described above for the plant sRNAs.

\section{SRNA Target Predictions and Degradome Analysis}

The wheat and $Z$. tritici sRNAs upregulated or highly expressed $(\mathrm{RPM} \geq 100)$ at 7 or 12 dpi were used to predict the target genes in wheat and $Z$. tritici transcriptome using psRNATarget (Dai et al., 2018). Default parameters from Schema V2 (2017 release) were used, but the expectation value was set at 4.5. Only the targeted genes that were significantly downregulated while the sRNAs showed upregulation or were constantly highly expressed were considered as the predicted target genes.

To confirm the cleavage of the predicted target genes, we generated modified PARE libraries with RNAs from the fungus (in vitro), infected wheat leaves (7 and $12 \mathrm{dpi}$ ), and mock leaves at the same time points. The samples for the modified PARE libraries were the same ones used for the small RNA sequencing (sRNA-seq) libraries. Poly (A)+ RNA was isolated from the RNA samples. Illumina TruSeq sequencing adapter was ligated to the 5'P ends of the degraded mRNA. Next, first-strand complementary DNA (cDNA) synthesis was performed using a N6 randomized primer. After RNA hydrolysis, the 3' Illumina TruSeq sequencing adapter was ligated to the $5^{\prime}$ ends of the antisense first-strand cDNA fragments. The $5^{\prime} \mathrm{cDNA}$ fragments were finally amplified with PCR using the Illumina TruSeq sequencing adapters as primers. The libraries were sequenced on an Illumina Next Seq 500 single end at $1 \times 75$ bp using a Truseq SBS kit v3-HS (Illumina, Inc. San Diego, CA, USA, NCBI-SRA project number SRP154808).

The raw sequencing data were trimmed to remove adapters and trimmed length $\geq 15$ using Trimmomatic- 0.36 (Bolger et al., 2014) with the settings "ILLUMINACLIP : TruSeq3-SE.fa:2:30:10 LEADING:10 TRAILING:10 SLIDINGWINDOW:4:15 MINLEN:15". The clean reads were analyzed using CleaveLand4 (Brousse et al., 2014) with the parameters “-c 3 -p 0.05 -r 0.7” for every possible condition. Only the results with a $P$ value under 0.05 and a minimum free energy (MFE) ratio higher than 0.7 were regarded as significant results. We only considered the degradome peaks in categories $0,1,2$, and 3 . These categories are described in Cleaveland 4.

\section{mRNA-Seq Sequencing and Analysis}

The microRNA sequencing (mRNA-seq) data from the wheat samples infected with $Z$. tritici were generated in a previous study (Palma-Guerrero et al., 2017), and they are available at the National Center for Biotechnology Information (NCBI) Short Read Archive (SRA) under the project SRP077418. The new mRNA-seq data from the 3D7 isolate grown in vitro and plant mocks at 7 and 12 dpi were generated in this study using Illumina HiSeq2500 at $2 \times 125$ bp (NCBI-SRA project number SRP154808). All the raw sequencing reads were trimmed using Trimmomatic- 0.36 as described before. The clean reads were aligned against wheat genome and $Z$. tritici genome separately using tophat v2.1.1 (Kim et al., 2013). HTSeq-count v0.6.1 was used to calculate the gene counts (Anders et al., 2015). We used
TMM implemented in Bioconductor EdgeR v3.12.0 to normalize the library sizes and gene expressions (Robinson et al., 2010; Robinson and Oshlack, 2010). Differentially expressed genes were defined using EdgeR. The Benjamin-Hochberg false discovery rate correction was used to adjust $P$ values based on the exact Fisher test (Robinson et al., 2010). The genes with a log fold change $\geq 1$ (or -1 or less) and adjusted $P$ value $<0.05$ were determined as the differentially expressed genes. The Gene Ontology (GO) analysis was performed with TopGO v2.32.0 (Alexa and Rahnenfuhrer, 2016).

\section{Northern Blot Analysis}

Total RNA from wheat leaves infected by $Z$. tritici at $12 \mathrm{dpi}$ and uninfected wheat leaves at the same time point were used for Northern blot. Of total RNA, $7.5 \mu \mathrm{g}$ was separated on $17.5 \%$ polyacrylamide-urea gels, electro-transferred to a HybondNX membrane (GE Healthcare), and cross-linked via 1-ethyl3-(3-dimethylaminopropyl) carbodiimide-mediated chemical cross-linking, as previously described (Bologna et al., 2018). Oligonucleotides were end-labeled by incubation with T4 PNK (Thermo Scientific) in the presence of $[\gamma-32 \mathrm{P}]$ dATP. Multiple small RNAs were hybridized on individual membranes by stripping twice with boiling $0.1 \%$ SDS and reprobing. The probe used for miRNA-uniq-1, miRNA-uniq-113, and miRNA-uniq-133 were: "GGGGAATGAAGCCTGG TCCGA," "GTGCTCACTCTCTTCTGTCA," and "TGGC ATACAGGGAGCCAGGCA," respectively. U6 was used as positive control.

\section{Quantification of Relative Gene Expression by Quantitative Real-Time RT-qPCR}

The transcription levels of four candidate genes predicted to be cleaved by sRNAs were analyzed using quantitative reverse transcription polymerase chain reaction (qRT-PCR). Six hundred nanograms of total RNA from leaves infected by $Z$. tritici at $12 \mathrm{dpi}$ and uninfected mock leaves was retrotranscribed by using the Revert First Strand cDNA Synthesis kit (Thermo Fisher) following the manufacturer's instructions. Primer efficiencies were determined by qPCR amplification of a serial dilution of genomic DNA. A melting analysis was performed at the end of the amplification to confirm single product amplification, and non-retrotranscribed RNA was used to discard DNA contamination in the RNA samples. For each candidate gene, three biological and three technical replications were used. Relative expression was calculated from the Ct values of the genes during infection compared to the mock samples using wheat $18 \mathrm{~s}$ rRNA gene for normalization.

\section{RESULTS}

\section{Z. tritici Produces sRNAs During Wheat Infection}

To explore the roles of RNAi in the wheat-Z. tritici pathosystem, we analyzed sRNA-seq libraries from samples of wheat leaves infected by $Z$. tritici after 7, 12, and $14 \mathrm{dpi}$. The corresponding 
wheat mock leaves at the same points and $Z$. tritici grown in vitro were used as controls. Three biological replicates were used for each condition. According to the description of the disease cycle (Rudd et al., 2015; Sanchez-Vallet et al., 2015), our dataset covered the latent period (7 dpi), transition period (12 dpi), and the early necrotrophic period (14 dpi) of the 3D7 strain disease cycle (Palma-Guerrero et al., 2017).

Reads with similarity to tRNAs, rRNAs, and snoRNAs of wheat and $Z$. tritici were filtered and the remaining reads were aligned against the wheat and the $Z$. tritici genome separately to distinguish the plant and fungal sRNA reads (Table 1). In total, we obtained 0.3-1.1 million fungal sRNA reads in the infected samples and 1.8-2.8 million fungal sRNA reads in the in vitro samples. Between 3.4 and 18.1 million plant sRNA reads were obtained in the infected samples and 1.9-6.5 million plant sRNA reads in the mock samples. The number of fungal sRNA reads increased during the infection process while the number of plant sRNA reads decreased, which is consistent with the disease progression on the infected leaves.

Using a customized bioinformatic analysis (see Methods section), we predicted 662 unique Z. tritici RNAs in total (Table S1) (Shahid and Axtell, 2014; Johnson et al., 2016). We considered all the major RNAs predicted by ShortStack as the fungal sRNAs. The most abundant fungal sRNAs were of 20 and $21 \mathrm{nt}$ in length (Figure 1A). The fungal sRNAs were originated across the fungal genome, including both core and accessory chromosomes, although most sRNAs came from chromosomes 1 and 3 (Figure 1B). Among the total number of fungal sRNAs, 160 were originated from gene exon regions, 19 were from gene intron regions, 343 were from transposable elements, and 212 were from the intergenic regions (Figure 1C). The size distribution and the chromosome of origin of these Z. tritici sRNAs are consistent with the previous study (Kettles et al., 2019). Besides, a similar proportion of nucleotides at $5^{\prime}$ end of the sRNAs was found in our analysis (Figure 2A).

\section{The Composition of Fungal sRNAs Changes During the Infection Cycle}

We analyzed the differentially expressed fungal sRNAs between infected samples and in vitro samples to identify the infectioninduced fungal sRNAs (Table S2). We detected that 66 fungal sRNAs were significantly upregulated in the infected samples compared to the in vitro samples (Figure 2A). These fungal sRNAs were considered as the infection-induced fungal sRNAs and therefore they may play important roles during plant infection. The number of induced fungal sRNAs was only 16 at $7 \mathrm{dpi}$, when the fungal pathogen was in the latent period and no symptoms were detected on the leaves. But this number increased to 30 at $12 \mathrm{dpi}$, when the first chlorotic symptoms appeared on the leaves, and 59 at $14 \mathrm{dpi}$, when necrotic lesions are clearly observed on the leaves (Palma-Guerrero et al., 2017). Besides, there were 174 fungal sRNAs significantly downregulated in the infection samples (Figure 2A).

The length and the nucleotide at $5^{\prime}$ end of the sRNAs are considered to determine to which AGO proteins are they binding, as it has been shown for Arabidopsis, and subsequently the sRNA final function (Mi et al., 2008). In our analysis, the length distributions of the differentially expressed fungal sRNAs between in vitro and infected samples were similar, being 20/21 nt (Figures $\mathbf{1 A}$ and $\mathbf{2 B}$ ). At the same time,

TABLE 1 | sRNA seq data summary.

\begin{tabular}{|c|c|c|c|c|c|c|}
\hline Conditions & Library ID & Total clean reads & Fungal sRNA reads & Fungal sRNA ratios (\%) & Plant sRNA reads & Plant sRNA ratios (\%) \\
\hline \multirow[t]{9}{*}{ Infection samples } & 3D7-7dpi-2 & $71,764,255$ & 339,262 & 0.47 & $18,186,405$ & 25.34 \\
\hline & 3D7-7dpi-8 & $75,365,538$ & 385,127 & 0.51 & $17,763,333$ & 23.57 \\
\hline & 3D7-7dpi-10 & $48,440,536$ & 306,834 & 0.63 & $9,039,173$ & 18.66 \\
\hline & 3D7-12dpi-2 & $65,969,573$ & 460,119 & 0.70 & $12,378,083$ & 18.76 \\
\hline & 3D7-12dpi-5 & $65,138,243$ & 653,052 & 1.00 & $10,109,163$ & 15.52 \\
\hline & 3D7-12dpi-6 & $45,790,107$ & 317,915 & 0.69 & $10,725,249$ & 23.42 \\
\hline & 3D7-14dpi-4 & $31,090,290$ & 901,839 & 2.90 & $3,783,930$ & 12.17 \\
\hline & 3D7-14dpi-5 & $34,372,386$ & 577,053 & 1.68 & $5,365,083$ & 15.61 \\
\hline & 3D7-14dpi-10 & $30,549,120$ & $1,108,689$ & 3.63 & $3,406,298$ & 11.15 \\
\hline \multirow[t]{3}{*}{ Fungal in vitro } & 3D7-vitro-1 & $16,547,092$ & $2,865,958$ & 17.32 & - & - \\
\hline & 3D7-vitro-2 & $12,318,911$ & $2,191,788$ & 17.79 & - & - \\
\hline & 3D7-vitro-3 & $9,515,263$ & $1,819,328$ & 19.12 & - & - \\
\hline \multirow[t]{9}{*}{ Plant mocks } & mock-7dpi-C1 & $17,166,095$ & - & - & $4,586,381$ & 26.72 \\
\hline & mock-7dpi-C2 & $21,565,083$ & - & - & $5,321,432$ & 24.68 \\
\hline & mock-7dpi-C3 & $24,379,228$ & - & - & $6,568,124$ & 26.94 \\
\hline & mock-12dpi-C1 & $20,048,211$ & - & - & $3,884,980$ & 19.38 \\
\hline & mock-12dpi-C2 & $23,466,121$ & - & - & $4,787,936$ & 20.40 \\
\hline & mock-12dpi-C4 & $14,114,494$ & - & - & $3,065,456$ & 21.72 \\
\hline & mock-14dpi-C1 & $8,993,244$ & - & - & $1,902,557$ & 21.16 \\
\hline & mock-14dpi-C2 & $28,168,941$ & - & - & $6,222,211$ & 22.09 \\
\hline & mock-14dpi-C4 & $11,895,554$ & - & - & $2,884,932$ & 24.25 \\
\hline
\end{tabular}

$s R N A$ ratios represent the percentage of fungal or plant $s R N A s$ among the total amount of clean reads. 

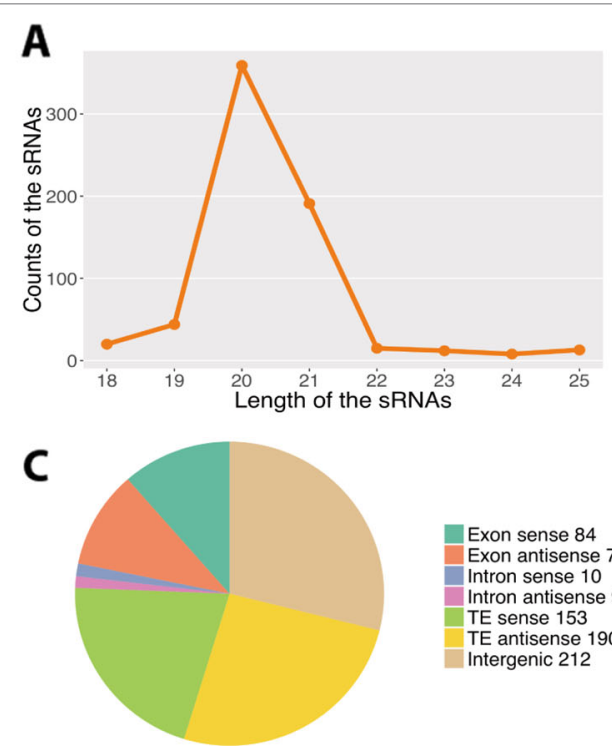

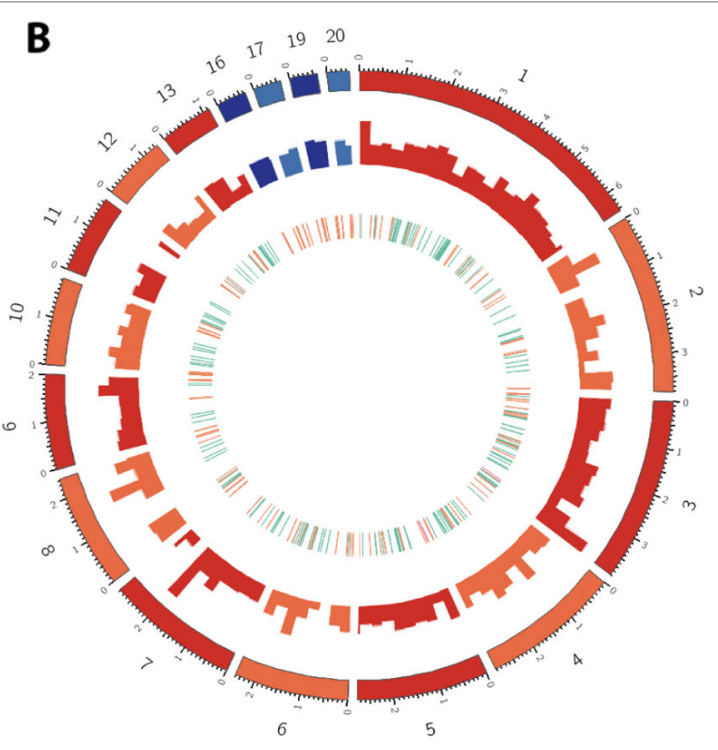

FIGURE 1 | Predictions of the fungal small RNAs (sRNAs). (A) Length distribution of the predicted fungal sRNAs. The $x$-axis indicates the lengths of the sRNAs and the $y$-axis indicates the counts of the sRNAs. (B) Distribution of the fungal sRNAs across the fungal genome. The outer layer shows the chromosomes of the fungal genome. The red colors are the core chromosomes and the blue colors are the accessory chromosomes. Accumulation of fungal sRNAs (log2-transformed) is shown in the middle layer. The inner layer shows the genomic elements where fungal sRNAs come from. The green ones reveal the sRNAs come from the gene region, the orange ones from transposable element (TE), and the pink ones from the overlap of gene and TE regions. (C) Origin of the fungal sRNAs. The different colors reveal the genomic elements where the fungal sRNAs come from. Sense means the sRNAs come from the same strand with the genomic elements while antisense means the opposite strand.

most of the fungal sRNAs shared uracil (U) at $5^{\prime}$ end, which have been shown to bind preferentially to AGO1 in Arabidopsis (Figure 2C). Interestingly, the proportion of $U$ at $5^{\prime}$ end changed dramatically, from $87 \%$ among all the sRNAs to $48 \%$ among the infection-induced sRNAs. Correspondingly, $23 \%$ of the infection-induced fungal sRNAs started with C, binding preferentially to AGO5 in Arabidopsis, and $16.7 \%$ started with A, binding preferentially to AGO2 and AGO3 in Arabidopsis (Figure 2C). This change indicates that there is different fungal RNA accumulation between growth in vitro and in planta and that the sRNAs induced during infection could have different functions from the sRNAs produced during in vitro growth, being part of different AGOs/sRNA complexes.

\section{Z. tritici SRNAs Were Not Detected to Cleave Wheat Transcripts}

The cross-kingdom RNAi between plants and fungal pathogens has been shown in different pathosystems (Zhang et al., 2011; Weiberg et al., 2013; Wang et al., 2016; Zhang et al., 2016; Wang et al., 2017). To investigate the presence of cross-kingdom RNAi in the wheat-Z. tritici pathosystem, we used the predicted fungal sRNAs to detect target genes in the wheat transcriptome. We found that 33 fungal sRNAs were either highly expressed (above $100 \mathrm{RPM}$ ) or upregulated at 7 or $12 \mathrm{dpi}$, which were predicted to target wheat genes. Among these targeted wheat genes, 139 were significantly downregulated during the infection (Table S3).

These 139 wheat genes were significantly enriched in chlorophyll-related processes and pigment biosynthesis (Figure 3A), which would affect photosynthesis, the leaf color, and the plant defense ability (Menzies et al., 2016). This suggests that fungal sRNAs may regulate plant chlorophyll-related genes to reduce the plant defense ability. Interestingly, these targeted wheat genes were enriched in "microtubule-based movement" biological process, which could affect the plant intracellular transport and secretion, and therefore affect the plant response to the pathogen infection (Lee et al., 2012). Besides these, fungal sRNAs were also predicted to target wheat receptor-like kinase (RLK) genes and resistance genes, which also suggests that fungal sRNAs were trying to disturb the plant defense system (Table S3).

Next, we performed a degradome analysis to validate the cleavage of the candidate targeted genes. Three conditions were used in this analysis: $Z$. tritici grown in vitro, wheat leaves infected by $Z$. tritici ( 7 and $12 \mathrm{dpi}$ ), and uninfected mock leaves at the same time points. To determine the quality of the degradome sequencing, we first analyzed several plant miRNAs that are already known to target plant genes. In our analysis, these plant miRNAs were predicted to target and cleave plant transcripts, as previously reported, which confirmed the good quality of the degradome sequencing. For example, miRNAuniq-21 (known as miR393) and miRNA-uniq-46 (known as miR156) were correctly found to cleave TIR1 genes and Squamosa promoter-binding-like (SPL) transcription factor genes, respectively, in our data (Figures 3B, C), as has been previously reported (Addo-Quaye et al., 2009).

In the degradome analysis, only one wheat gene, TraesCS3B01G136500, was found to be cleaved and downregulated by fungal sRNA56 at 7 dpi (Figure 3D). This wheat gene encodes 

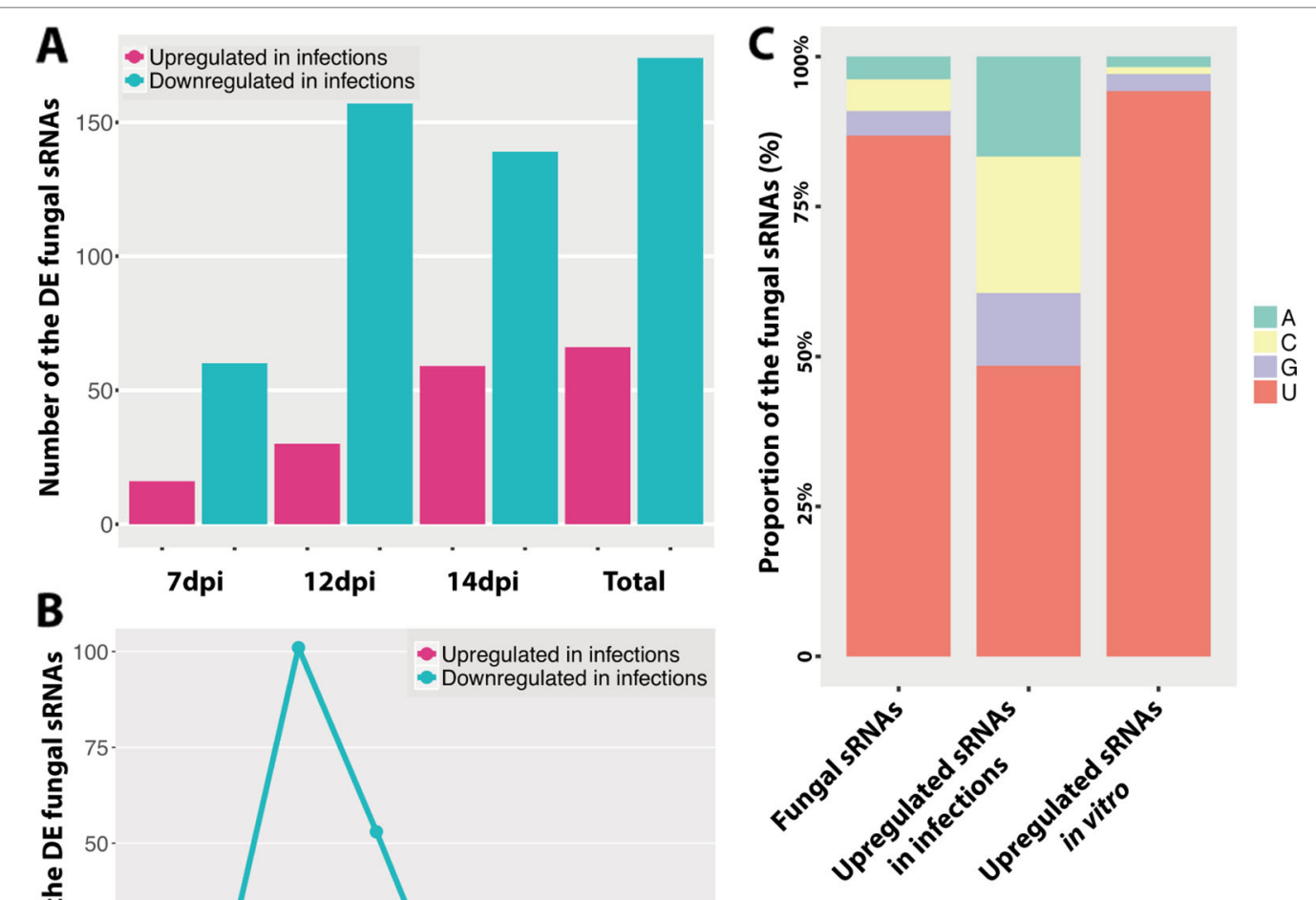

FIGURE 2 | Differentially expressed fungal small RNAs (sRNAs) during the infection cycle. (A) Number of the differentially expressed (DE) fungal sRNAs during the infection cycle. "Total" indicates the total number of DE fungal sRNAs across all time points. (B) Length distributions of the DE fungal sRNAs. (C) Frequency of nucleotides at $5^{\prime}$ end of the fungal sRNAs.

a "Remodeling and spacing factor 1 (RSF-1)" protein, which is known to be involved in DNA double-strand break repair in mammals (Pessina and Lowndes, 2014). SRNA56 was induced during infection, but being low expressed (Figure 3E). Additionally, the significantly reduced target, TraesCS3B01G136500, was constantly very low expressed (below 3 RPKM) and the degradome coverage of the cleaved transcripts was also low, which raises doubt about it being a real target (Figures 3D, F). These degradome sequencing results support the recent findings from Kettles et al. showing that the IPO323 strain of $Z$. tritici could not cleave wheat transcripts during infection (Kettles et al., 2019).

We also investigated the RNAi in $Z$. tritici during the disease cycle. In total, 39 fungal sRNAs were predicted to target and downregulate the fungal own genes during the infection (Table S4). But none of these genes were detected to be cleaved in the degradome sequencing. Finally, we analyzed the expressions of genes involved in RNAi in Z. tritici. There is only one Dicer gene in the $Z$. tritici genome (g9428 from the 3D7 genome), which is consistent across five different sequenced $Z$. tritici genomes (Goodwin et al., 2011; Plissonneau et al., 2016; Plissonneau et al., 2018). Surprisingly, this Dicer gene was significantly downregulated in planta compared with in vitro (Figure 4A). Additionally, the sRNAs were more abundant in vitro than in planta, which could be caused by the low expression of the fungal Dicer gene (Figure 4B). There are four AGO genes in the $Z$. tritici genome (g4308, g1839, g10449, and g2094 of the 3D7 genome), among which the AGO gene g2094 was not expressed in any condition tested. We as well did not find any significant upregulation of AGO genes in Z. tritici during infection (Figure 4A). The downregulation pattern of RNAi-related genes in $Z$. tritici also indicates their unimportant roles during wheat infection.

\section{Wheat Regulates sRNAs in Response to Fungal Infections}

We used the same dataset to identify the wheat sRNAs and to study the roles of wheat sRNAs in the disease cycle. In total, we identified 158 wheat miRNAs and 1,120 wheat siRNA loci (Table S5). By blasting these predicted miRNAs against the known wheat miRNAs, we found 69 miRNAs that were already annotated in the wheat genome. The other 89 miRNAs were 


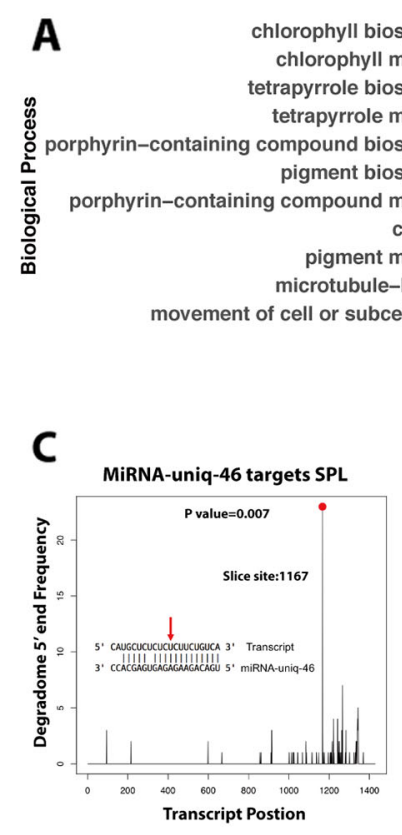

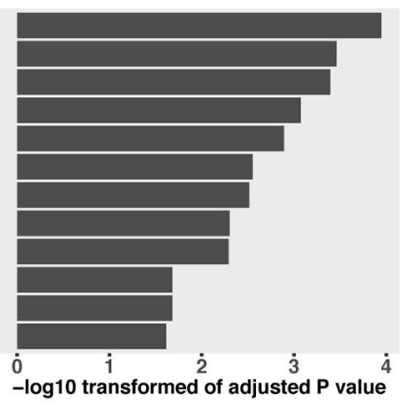

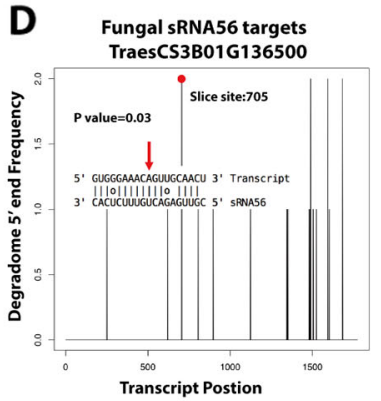

E
B
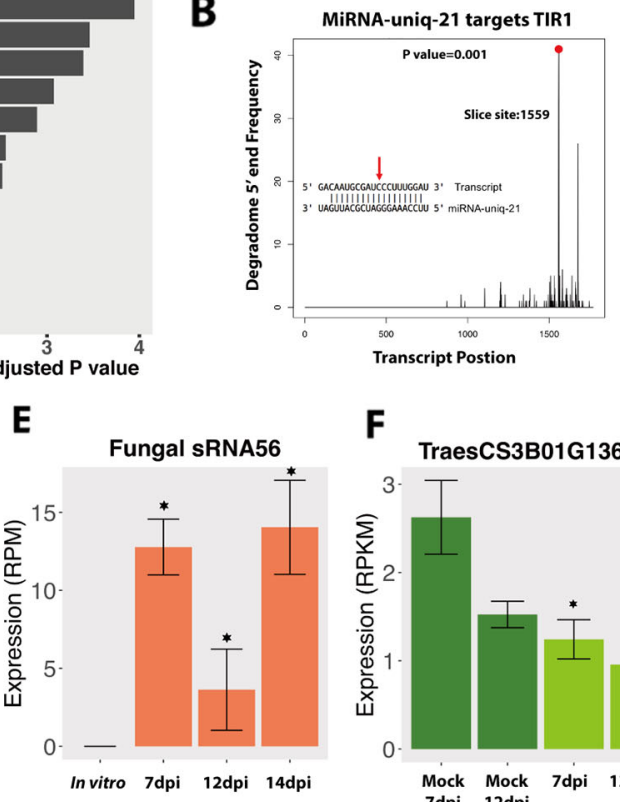

$\mathbf{F}$

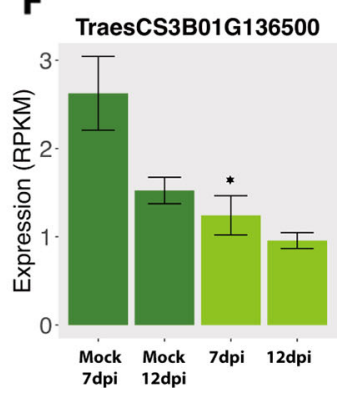

FIGURE 3 | Degradome analysis of the fungal small RNAs (sRNAs). (A) GO enrichment of biological process of wheat genes that were downregulated and predicted to be targeted by fungal-induced sRNAs during the infections. (B, C) Positive controls of degradome sequencing results. The $x$-axis indicates the position of the target transcript and the $y$-axis indicates the degraded frequency of the target transcript. The red point and red arrow indicate the slice site from the transcript and the sRNA. The $P$ value indicates the significance of the target. (D) Degradome results of fungal sRNA56 targeting plant gene. (E, F) Expressions of fungal sRNA56 and its target gene in plant. Asterisk indicates that the sRNA or the target genes are differentially expressed compared to the in vitro or the mock conditions, respectively.
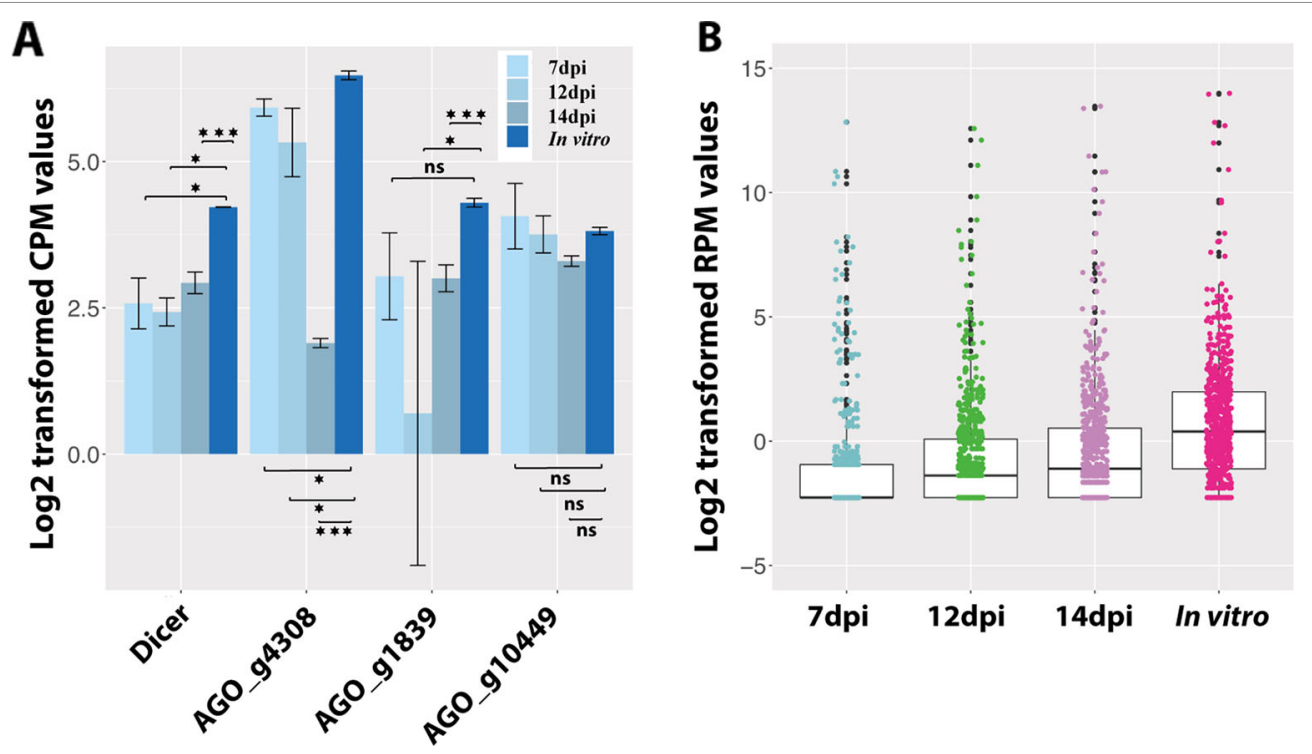

FIGURE 4 | Fungal small RNAs (sRNAs) are more abundant in vitro than in planta. (A) Expression of the fungal Dicer gene and three AGO genes. One asterisk indicates that the adjusted $P$ value is under 0.05 and three asterisks indicate that the $P$ value is under 0.001 . $n s$ indicates no significant difference. (B) Average expression of all the fungal SRNAs in planta and in vitro.

considered as novel wheat miRNAs (Table S5). Most wheatpredicted miRNAs were $21 \mathrm{nt}$ in length, consistent with previous studies (Figure 5A) (Rajagopalan et al., 2006; Axtell, 2013). The majority of these plant miRNAs start with $U$ at $5^{\prime}$ end (Figure 5B), which should favor binding the plant AGO1 proteins (Mi et al., 2008). Unlike the miRNAs, most of the wheat siRNAs were $24 \mathrm{nt}$ in length and started with A (Figures 5A, B). These 24-nt siRNAs have been shown to act by $\mathrm{RdDM}$ in Arabidopsis 

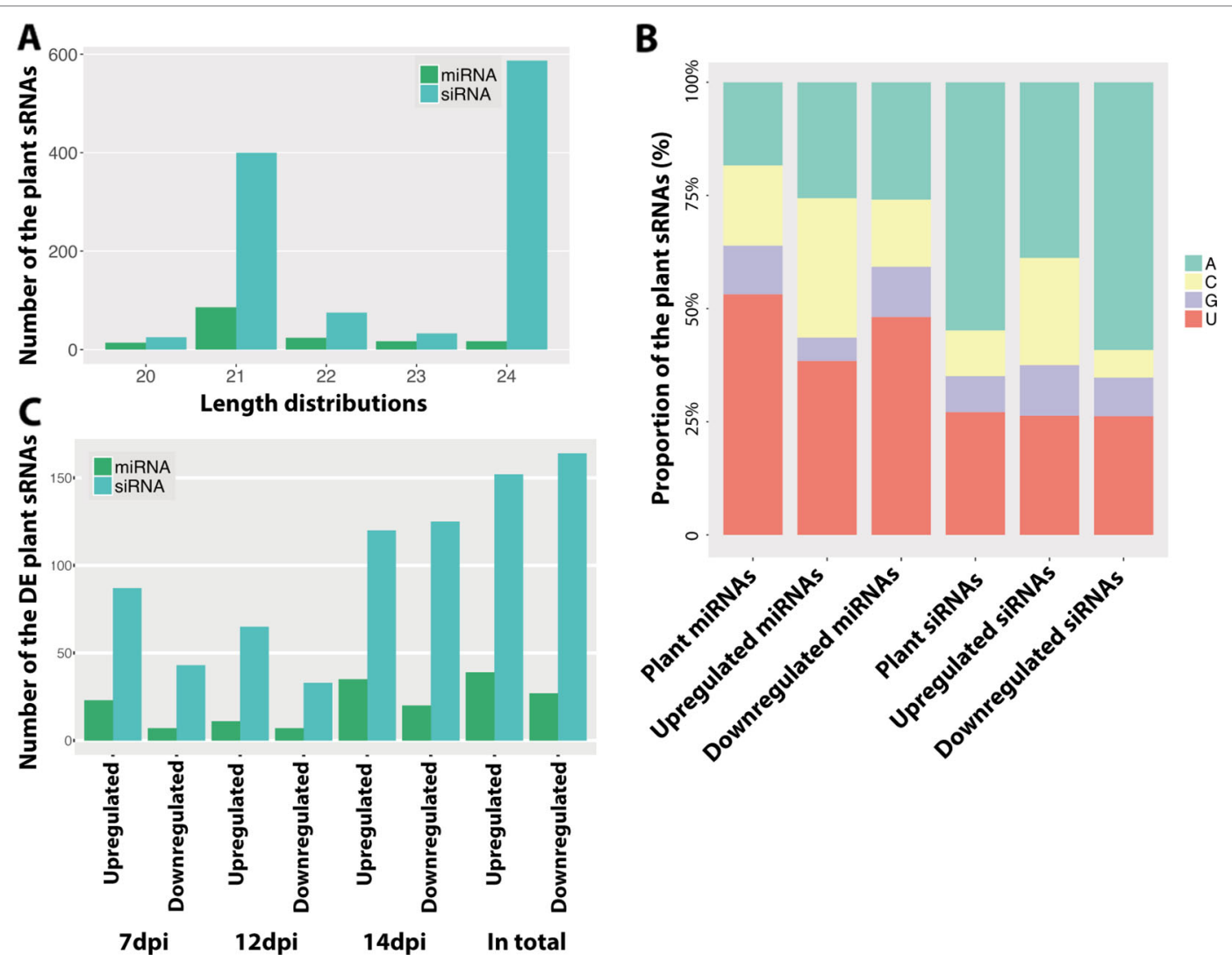

FIGURE 5 | Predicted plant small RNAs (sRNAs). (A) Length distribution of the predicted plant sRNAs. (B) Frequency of nucleotides at $5^{\prime}$ end of plant sRNAs. Different colors indicate different nucleotides. (C) Number of the differentially expressed sRNAs between infections and mocks. Upregulated indicates the sRNAs are upregulated in the infections and Downregulated indicates the sRNAs are downregulated in the infections.

(Matzke and Mosher, 2014), being preferentially loaded in AGO4 (Mi et al., 2008).

Plants can regulate the sRNAs, especially miRNAs, to respond to pathogens, which is thought to be a fast and efficient immune response (Fei et al., 2013). In order to determine if the infection induces variations in sRNA accumulation, we compared the expression of all the plant sRNAs between infected and mock samples at every time point. Like for the fungus, the infectioninduced plant sRNAs also exhibited different frequencies of nucleotides at $5^{\prime}$ end (Figure 5B), which suggests a different sRNA production during infections.

We found that 191 plant sRNAs were significantly upregulated in the infections, among which 39 were miRNAs (15 novel miRNAs) and 152 were siRNAs (Figure 5C and Table S6). Among these induced or extremely highly expressed (RPM above 100) plant sRNAs, 176 sRNAs (47 miRNAs and 129 siRNAs) were predicted to target and silence 690 wheat genes (Table S7). Interestingly, 20 (3\%) of these candidates targeted genes were resistance genes and 77 (11.2\%) were RLKs, which are considered to play central roles in plant immunity. Besides, 12 (2\%) genes encode ABC transporters and 11 (1.5\%) genes encode auxin response factor, which are also genes involved in the plant immune system (Figure 6A).
We confirmed the cleavage of three transcripts among these 690 genes (Figures 6B-D). MiRNA-uniq-133 (miRNA160 family) was detected to silence TraesCS6A01G222300.1, which encodes an auxin response factor (Figures 6B and S1). Two SPL genes were negatively regulated by miRNA-uniq-113 (belonging to miRNA156 family), which was upregulated at 7 and $12 \mathrm{dpi}$ (Figures 6C, D and S1). The expressions of these two miRNAs were confirmed by Northern blot analysis (Figure S2). The downregulation of these target genes during the fungal infection was confirmed both by RNA-seq data and qPCR experiments (Figures 6E and S1).

In plant-pathogen interactions, plants can as well export miRNAs into pathogen cells to influence pathogen virulence (Wang et al., 2016). We predicted 190 wheat sRNAs that were upregulated during the infections or extremely highly expressed (RPM above 100) and could target and downregulate 1,115 fungal genes (Table S8). Interestingly, these targeted fungal genes were significantly enriched in "vesicle-mediated transport" GO term (Figure 7). We detected that two of these 1,115 fungal genes could be cleaved by wheat sRNAs (Figure S3). One gene is g1915, which encodes an antibiotic biosynthesis monooxygenase domain $(\mathrm{ABM})$ protein. The other gene is g9791, which encodes FAD-dependent oxidoreductase. These 

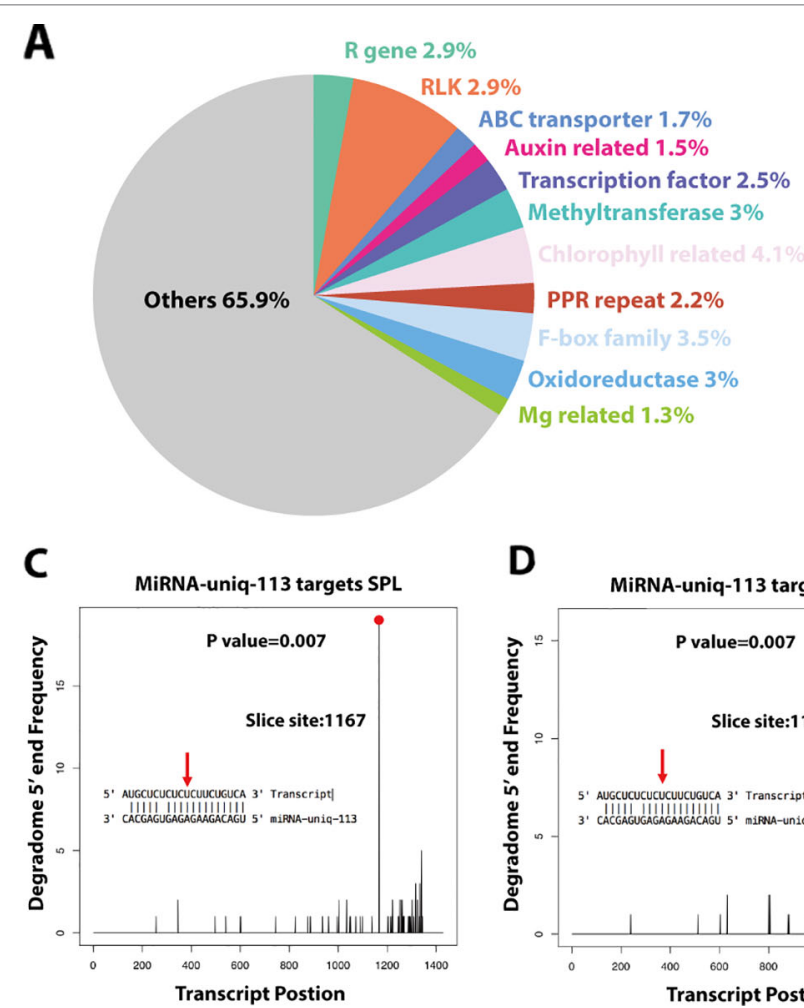

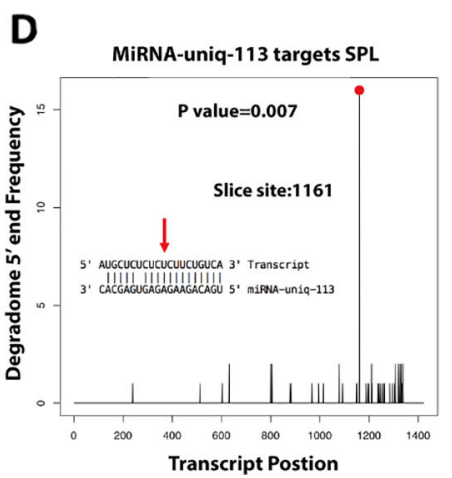

B

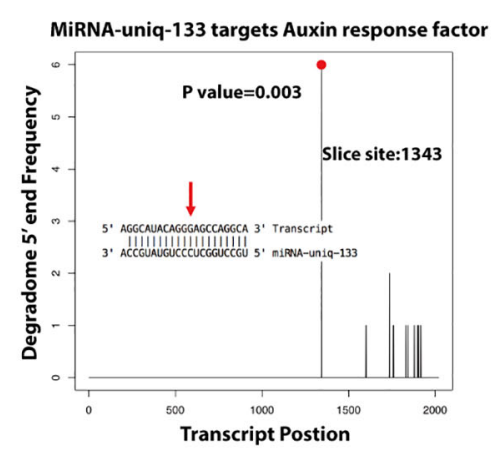

E

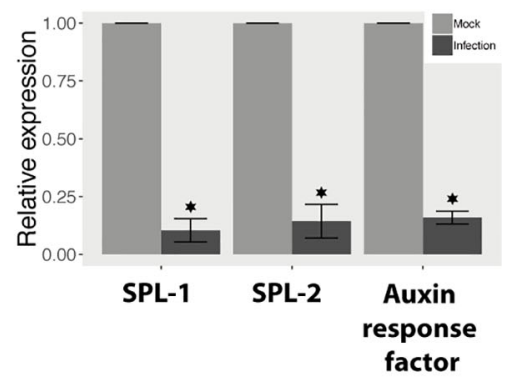

FIGURE 6 | Wheat induces small RNAs (sRNAs) to regulate wheat genes as an immune response against $Z$. tritici. (A) Functions of the wheat genes, which were downregulated and predicted to be targeted by wheat-induced sRNAs. (B-D) Degradome analysis of the wheat genes targeted by miRNA-uniq-133 and miRNAuniq-113. (E) Relative expression of miRNA-133 and miRNA-113 targeted genes in infections compared to mocks. Each value represents the average of three biological replicates, with three technical replicates per sample. Asterisk indicates significant downregulation during fungal infection $(P$ value under 0.05$)$.

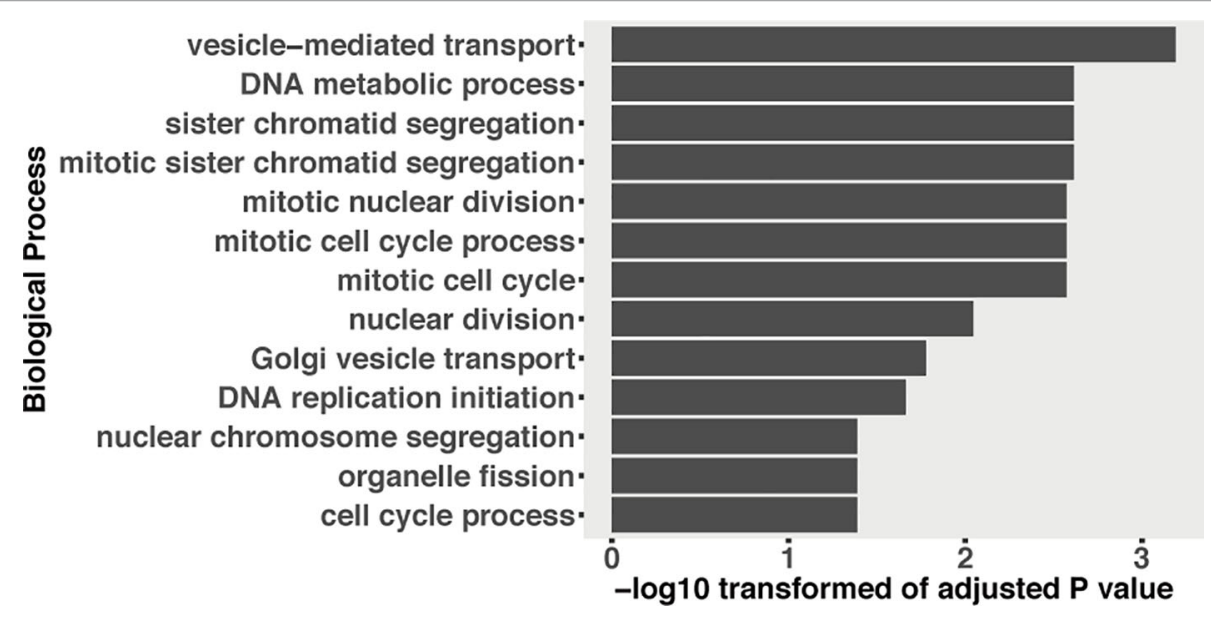

FIGURE 7 | GO enrichment of fungal genes, which were downregulated and predicted to be targeted by induced plant sRNAs during the infection.

two fungal genes were downregulated at $12 \mathrm{dpi}$ (only significant for g1915) and recovered their expressions from 14 dpi (Figure S3). However, it has to be noted that the degradome coverage over those genes was very low and the slice site of the g1915 transcript belongs to category 3 (Figure S3), which suggests that it is not a real target.

\section{DISCUSSION}

In this study, we combined sRNA-seq, mRNA-seq, and degradome sequencing from samples covering key stages during the infection cycle to analyze the role of sRNAs in the wheat and Z. tritici pathosystem. We found that both $Z$. tritici 
and wheat sRNAs do not play important roles in a crosskingdom manner, as shown by degradome sequencing, and supports the recently published findings for the $Z$. tritici sRNAs (Kettles et al., 2019). These two independent studies used two different $Z$. tritici strains (IPO323 and 3D7) and two different wheat cultivars (Bobwhite and Drifter) and got the same findings, indicating that cross-kingdom RNAi may not play important roles in the wheat and $Z$. tritici interaction. Additionally, we also analyzed wheat sRNAs during the disease cycle and we show that wheat can regulate wheat sRNAs in response to $Z$. tritici infections.

\section{RNAi Does not Play Important Roles in Z. tritici During Wheat Infection}

In this work, we predicted $Z$. tritici sRNAs during infection cycle. Interestingly, even at $7 \mathrm{dpi}$, when the fungus was in latent period and no symptoms are detected on wheat leaves, we still detected fungal sRNAs. The concentrations and expressions of $Z$. tritici sRNAs were lower at 7 dpi compared with the other time points, probably due to the low fungal biomass at this stage. Despite this, we found induced $Z$. tritici sRNAs at 7 dpi too. Fungal sRNAs can hijack the plant host RNAi machinery to silence plant defense-related genes and facilitate fungal infection (Weiberg et al., 2013; Wang et al., 2017). However, we could not find any cleavage of wheat transcripts caused by the fungal sRNAs in the degradome sequencing. We only detected one wheat gene, encoding the RSF-1 protein, in the degradome data, which was predicted to be cleaved by fungal sRNA56. But the low expressions of sRNA56 and the low frequency of the cleaved transcript from the target gene suggested it was not a real target, at least in the canonical way (Figures 3D-F).

We also predicted 39 fungal sRNA that could regulate fungal genes in planta. But none of these targeted fungal genes were detected to be cleaved in the degradome sequencing. The fungal Dicer gene was barely expressed in the infections, which led to a decrease of sRNA genesis by the fungus (Figure 4). This is supported by the lower expressions of sRNAs observed in planta than in vitro. In addition, deletion of the $Z$. tritici Dicer gene has been found to have no effect on fungal virulence (Kettles et al., 2019). These results, together with our findings, suggest minor roles of the $Z$. tritici Dicer for fungal virulence. These data suggest that fungal sRNAs are not cleaving gene transcripts during the wheat $-Z$. tritici interaction.

These results are consistent with the recent study by Kettles et al. (2019), in which they show that Z. tritici sRNAs could not silence wheat genes in a cross-kingdom manner during infection. In these two independent studies, two different $Z$. tritici strains have been used to infect two different wheat cultivars, obtaining the same results. Additionally, our degradome sequencing data support the findings validated by molecular studies in the previous study (Kettles et al., 2019), confirming the unimportant roles of $Z$. tritici sRNAs in cross-kingdom RNAi.

However, even if the $Z$. tritici sRNAs do not play a direct role in the interaction with wheat, they may play a role at specific developmental stages of the fungus, as has been shown for other plant pathogenic fungi (Kim et al., 2015; Raman et al.,
2017; Zeng et al., 2018). Also, we cannot discard the possibility that $Z$. tritici sRNAs may be acting at the transcriptional or translational level.

\section{Wheat sRNAs are Involved in the Response to Z. tritici Infection}

Previous studies have reported that plants can employ sRNAs as a rapid defense response against pathogens. These plant sRNAs could regulate auxin-related genes, MYB transcription factors, and as well $\mathrm{R}$ genes in plant (Achard et al., 2007; Chen et al., 2007; Navarro et al., 2008; Grant and Jones, 2009; Zhai et al., 2011; Shivaprasad et al., 2012; Fei et al., 2013; Samad et al., 2017).

Here, we found that 191 wheat sRNAs were induced during fungal infection, the composition of which were quite different from the plant sRNAs expressed in the mocks (Figure 5B). These induced wheat sRNAs were predicted to target and silence 690 wheat genes (Table S7), including $\mathrm{R}$ genes, RLKs, ABC transporters, and auxin response factors, which are known to play central roles in the plant immunity (Figure 6A). Besides, many transcription factor genes were also predicted to be targeted by these induced sRNAs, including SPL transcription factors, F-box domain proteins, MYB transcription factors, and basic/helix-loop-helix (bHLH) proteins (Figure 6A). We confirmed the cleavage of three transcripts, which encode on auxin response factor and two SPL proteins. (Figures 6, S1 and S2). The induced sRNA-mediated auxin-related gene here is consistent with the response observed during plant infection by bacterial pathogens (Navarro et al., 2008). When plants are under biotic stress, sRNAs are induced to regulate transcription factors as an immune response (Tsuda and Somssich, 2015). Here, we confirmed that SPL transcription factors are mainly involved in this sRNA-mediated immune response. Thus, we show clear evidence that wheat will induce a group of specific sRNAs to regulate wheat genes as an immune response to $Z$. tritici.

In HIGS, plants can transport RNA modules and target pathogen genes to reduce pathogen virulence (Nowara et al., 2010; Baulcombe, 2015). It has been proven recently that plants use extracellular vesicles to send sRNAs into fungal cells (Cai et al., 2018). These finding suggest that the RNA molecules can be transported to fungal pathogens naturally. We predicted 1,115 fungal genes that could be targeted by 190 wheat sRNAs. These wheat sRNAs were either upregulated or extremely highly expressed during the infections (Table S8). Interestingly, the targeted fungal genes were enriched in the "vesicle-mediated transport" GO term (Figure 7). Fungal extracellular vesicles (EVs) are essential to transport proteins, glycan pigments, nucleic acids, and lipids (Rodrigues et al., 2015; Rodrigues and Casadevall, 2018). Fungal EVs are also responsible to pathogenesis during the infection (Rodrigues et al., 2007; Albuquerque et al., 2008; Rodrigues et al., 2008; Vallejo et al., 2011; Vargas et al., 2015), including mediating the transport of virulent effectors into plant cells (Rodrigues et al., 2008). A recent study also proved that plant miRNAs can be transported by EVs into fungal cells to affect fungal virulence (Cai et al., 2018). Here, our results suggest that wheat sRNAs may target fungal EV- 
related genes to interfere fungal virulence. However, we only detected two fungal genes that were cleaved by the wheat sRNAs (Figure S3). Fungal g1915, which encodes an ABM protein, involved in diverse biological processes, including metabolism, transcription, translation, and biosynthesis of secondary metabolites, was cleaved by plant siRNA180. The other one is fungal g9791, which encodes FAD-dependent oxidoreductase, which was targeted by plant miRNA-uniq- 21 . These two fungal genes play fundamental roles in fungal growth and they are downregulated at the beginning of the disease cycle. However, these two fungal genes were not completely silenced, and the expression of these two genes was recovered at $14 \mathrm{dpi}$, when the fungus started the necrotrophic growth phase. But as we discussed before, the degradome results did not provide support for the cleavage of the transcripts from these two genes. These results suggest that wheat is not able to use sRNAs to silence fungal genes. These results are supported by the finding that dsRNAs generated from RNA virus vectors in planta are not effective in triggering gene silencing in $Z$. tritici during the fungal infection (Kettles et al., 2019).

In conclusion, our results support the recent finding that there is no natural cross-kingdom RNAi causing the cleavage of gene transcripts between these two interacting species. However, we found that wheat can use wheat sRNAs to regulate the plant defenses during $Z$. tritici infection. These findings contribute to improve our understanding of the hidden interactions in this pathosystem.

\section{DATA AVAILABILITY STATEMENT}

All the raw sequencing data were deposited into the NCBI Short Read Archive under the accession number SRP154808.

\section{REFERENCES}

Achard, P., Baghour, M., Chapple, A., Hedden, P., Van Der Straeten, D., Genschik, P., et al. (2007). The plant stress hormone ethylene controls floral transition via DELLA-dependent regulation of floral meristem-identity genes. Proc. Natl. Acad. Sci. U.S.A. 104, 6484-6489. doi: 10.1073/pnas.0610717104

Addo-Quaye, C., Snyder, J. A., Park, Y. B., Li, Y. F., Sunkar, R., and Axtell, M. J. (2009). Sliced microRNA targets and precise loop-first processing of MIR319 hairpins revealed by analysis of the Physcomitrella patens degradome. RNA 15, 2112-2121. doi: 10.1261/rna.1774909

Albuquerque, P. C., Nakayasu, E. S., Rodrigues, M. L., Frases, S., Casadevall, A., ZancopeOliveira, R. M., et al. (2008). Vesicular transport in Histoplasma capsulatum: an effective mechanism for trans-cell wall transfer of proteins and lipids in ascomycetes. Cell Microbiol. 10, 1695-1710. doi: 10.1111/j.1462-5822.2008.01160.x

Alexa, A., and Rahnenfuhrer, J. (2016). topGO: Enrichment analysis for gene ontology. R package version 2.32.0.

Allen, E., Xie, Z., Gustafson, A. M., and Carrington, J. C. (2005). microRNAdirected phasing during trans-acting siRNA biogenesis in plants. Cell 121, 207221. doi: $10.1016 /$ j.cell.2005.04.004

Anders, S., Pyl, P. T., and Huber, W. (2015). HTSeq-a Python framework to work with high-throughput sequencing data. Bioinformatics 31, 166-169. doi: 10.1093/bioinformatics/btu638

Appels, R., Eversole, K., Feuillet, C., Keller, B., Rogers, J., Stein, N., et al. (2018). Shifting the limits in wheat research and breeding using a fully annotated reference genome. Science 361 (6403), eaar7191. doi: 10.1126/science.aar7191

\section{AUTHOR CONTRIBUTIONS}

$\mathrm{XM}$, JW, and JP-G conceived and designed experiments. XM was responsible for the bioinformatics analysis. JW, XM, and JP-G were responsible for the experimental validations. XM, and JP-G wrote the paper.

\section{FUNDING}

$\mathrm{XM}$ is funded by a PSC-Syngenta $\mathrm{PhD}$ fellowship. Laboratory facilities were provided by the Genetic Diversity Center (GDC) of ETH Zurich.

\section{ACKNOWLEDGMENTS}

sRNA and mRNA sequencing was performed at the Functional Genomics Center Zurich (FGCZ). The degradome sequencing was performed by Vertis Biotechnology AG (Germany). We thank Carolina S. Francisco for providing the RNA samples of the fungus in vitro used as control. We are also very grateful to Dr. Nicolas Bologna, Dr. Thomas Grentzinger, and Dr. Alexis Sarazin for their suggestions and for the interesting scientific discussions related to this project. This manuscript has been released as a preprint at BioRxiv (Ma et al., 2018a).

\section{SUPPLEMENTARY MATERIAL}

The Supplementary Material for this article can be found online at: https://www.frontiersin.org/articles/10.3389/fpls.2019. 01669/full\#supplementary-material

Axtell, M. J. (2013). Classification and comparison of small RNAs from plants. Annu. Rev.PlantBiol.Vol6464,137-159.doi: 10.1146/annurev-arplant-050312-120043 Axtell, M. J., and Meyers, B. C. (2018). Revisiting criteria for plant microRNA annotation in the era of big data. Plant Cell 30, 272-284. doi: 10.1105/tpc.17.00851

Baulcombe, D. C. (2015). VIGS, HIGS and FIGS: small RNA silencing in the interactions of viruses or filamentous organisms with their plant hosts. Curr. Opin. Plant Biol. 26, 141-146. doi: 10.1016/j.pbi.2015.06.007

Bolger, A. M., Lohse, M., and Usadel, B. (2014). Trimmomatic: a flexible trimmer for Illumina sequence data. Bioinformatics 30, 2114-2120. doi: 10.1093/ bioinformatics/btu170

Bologna, N. G., and Voinnet, O. (2014). The diversity, biogenesis, and activities of endogenous silencing small RNAs in Arabidopsis. Annu. Rev. Plant Biol. 65, 473-503. doi: 10.1146/annurev-arplant-050213-035728

Bologna, N. G., Iselin, R., Abriata, L. A., Sarazin, A., Pumplin, N., Jay, F., et al. (2018). Nucleo-cytosolic Shuttling of argonautel prompts a revised model of the plant microRNA pathway. Mol. Cell 69709-719, e705. doi: 10.1016/ j.molcel.2018.01.007

Borges, F., and Martienssen, R. A. (2015). The expanding world of small RNAs in plants. Nat. Rev. Mol. Cell Biol. 16, 727-741. doi: 10.1038/nrm4085

Brousse, C., Liu, Q., Beauclair, L., Deremetz, A., Axtell, M. J., and Bouche, N. (2014). A non-canonical plant microRNA target site. Nucleic Acids Res. 42, 5270-5279. doi: 10.1093/nar/gku157

Cai, Q., Qiao, L., Wang, M., He, B., Lin, F. M., Palmquist, J., et al. (2018). Plants send small RNAs in extracellular vesicles to fungal pathogen to silence virulence genes. Science. 360 (6393), 1126-1129. doi: 10.1126/science.aar4142 
Camacho, C., Coulouris, G., Avagyan, V., Ma, N., Papadopoulos, J., Bealer, K., et al. (2009). BLAST plus: architecture and applications. BMC Bioinf. 10, 421. doi: 10.1186/1471-2105-10-421

Carthew, R. W., and Sontheimer, E. J. (2009). Origins and Mechanisms of miRNAs and siRNAs. Cell 136, 642-655. doi: 10.1016/j.cell.2009.01.035

Chang, S. S., Zhang, Z. Y., and Liu, Y. (2012). RNA interference pathways in fungi: mechanisms and functions. Annu. Rev. Microbiol. Vol 66 66, 305-323. doi: 10.1146/annurev-micro-092611-150138

Chen, Z., Agnew, J. L., Cohen, J. D., He, P., Shan, L., Sheen, J., et al. (2007). Pseudomonas syringae type III effector AvrRpt2 alters Arabidopsis thaliana auxin physiology. Proc. Natl. Acad. Sci. U.S.A. 104, 20131-20136. doi: 10.1073/pnas.0704901104

Creasey, K. M., Zhai, J., Borges, F., Van Ex, F., Regulski, M., Meyers, B. C., et al. (2014). miRNAs trigger widespread epigenetically activated siRNAs from transposons in Arabidopsis. Nature 508, 411-415. doi: 10.1038/nature13069

Dai, X., Zhuang, Z., and Zhao, P. X. (2018). psRNATarget: a plant small RNA target analysis server (2017 release). Nucleic Acids Res. 46, W49-W54. doi: 10.1093/nar/gky316

Fei, Q., Xia, R., and Meyers, B. C. (2013). Phased, secondary, small interfering RNAs in posttranscriptional regulatory networks. Plant Cell 25, 2400-2415. doi: $10.1105 /$ tpc.113.114652

Felippes, F. F., and Weigel, D. (2009). Triggering the formation of tasiRNAs in Arabidopsis thaliana: the role of microRNA miR173. EMBO Rep. 10, 264-270. doi: 10.1038/embor.2008.247

Francisco, C. S., Ma, X., Zwyssig, M. M., Mcdonald, B. A., and Palma-Guerrero, J. (2019). Morphological changes in response to environmental stresses in the fungal plant pathogen Zymoseptoria tritici. Sci. Rep. 9642 (9). doi: 10.1101/372078

Goodwin, S. B., M'barek S, B., Dhillon, B., Wittenberg, A. H., Crane, C. F., Hane, J. K., et al. (2011). Finished genome of the fungal wheat pathogen Mycosphaerella graminicola reveals dispensome structure, chromosome plasticity, and stealth pathogenesis. PloS Genet. 7, e1002070. doi: 10.1371/journal.pgen.1002070

Gordon, A., and Hannon, G. (2010). Fastx-toolkit. FASTQ/A short-reads preprocessing tools (unpublished).

Grant, M. R., and Jones, J. D. (2009). Hormone (dis)harmony moulds plant health and disease. Science 324, 750-752. doi: 10.1126/science.1173771

Griffiths-Jones, S., Grocock, R. J., Van Dongen, S., Bateman, A., and Enright, A. J. (2006). miRBase: microRNA sequences, targets and gene nomenclature. Nucleic Acids Res. 34, D140-D144. doi: 10.1093/nar/gkj112

Griffiths-Jones, S., Saini, H. K., Van Dongen, S., and Enright, A. J. (2008). miRBase: tools for microRNA genomics. Nucleic Acids Res. 36, D154-D158. doi: 10.1093/nar/gkm952

Griffiths-Jones, S. (2004). The microRNA registry. Nucleic Acids Res. 32, D109D111. doi: 10.1093/nar/gkh023

Guleria, P., Mahajan, M., Bhardwaj, J., and Yadav, S. K. (2011). Plant small RNAs: biogenesis, mode of action and their roles in abiotic stresses. Genomics Proteomics Bioinf. 9, 183-199. doi: 10.1016/S1672-0229(11)60022-3

Hardwick, N. V., Jones, D. R., and Slough, J. E. (2001). Factors affecting diseases of winter wheat in England and Wales, 1989-98. Plant Pathol. 50, 453-462. doi: 10.1046/j.1365-3059.2001.00596.x

Hutvagner, G., and Simard, M. J. (2008). Argonaute proteins: key players in RNA silencing. Nat. Rev. Mol. Cell Biol. 9, 22-32. doi: 10.1038/nrm2321

Johnson, N. R., Yeoh, J. M., Coruh, C., and Axtell, M. J. (2016). Improved placement of multi-mapping small RNAs. G3 (Bethesda) 6, 2103-2111. doi: $10.1534 / \mathrm{g} 3.116 .030452$

Kamthan, A., Chaudhuri, A., Kamthan, M., and Datta, A. (2015). Small RNAs in plants: recent development and application for crop improvement. Front. Plant Sci. 6, 208. doi: 10.3389/fpls.2015.00208

Kersey, P. J., Allen, J. E., Allot, A., Barba, M., Boddu, S., Bolt, B. J., et al. (2018). Ensembl genomes 2018: an integrated omics infrastructure for non-vertebrate species. Nucleic Acids Res. 46, D802-D808. doi: 10.1093/nar/gkx1011

Kettles, G. J., Hofinger, B. J., Hu, P., Bayon, C., Rudd, J. J., Balmer, D., et al. (2019). sRNA profiling combined with gene function analysis reveals a lack of evidence for cross-kingdom rnai in the wheat - Zymoseptoria tritici pathosystem. Front. Plant Sci. 10, 892. doi: 10.3389/fpls.2019.00892

Kim, D., Pertea, G., Trapnell, C., Pimentel, H., Kelley, R., and Salzberg, S. L. (2013). TopHat2: accurate alignment of transcriptomes in the presence of insertions, deletions and gene fusions. Genome Biol. 14, R36. doi: 10.1186/gb-2013-14-4-r36

Kim, H. K., Jo, S. M., Kim, G. Y., Kim, D. W., Kim, Y. K., and Yun, S. H. (2015). A large scale functional analysis of putative genes of mating type loci provides insight into the regulation of sexual development of the cereal pathogen fusarium graminearum. PloS Genet. 11 (9), e1005486. doi: 10.1371/journal.pgen.1005486

Kozomara, A., and Griffiths-Jones, S. (2011). miRBase: integrating microRNA annotation and deep-sequencing data. Nucleic Acids Res. 39, D152-D157. doi: $10.1093 /$ nar/gkq1027

Kozomara, A., and Griffiths-Jones, S. (2014). miRBase: annotating high confidence microRNAs using deep sequencing data. Nucleic Acids Res. 42, D68-D73. doi: 10.1093/nar/gkt1181

Langmead, B., Trapnell, C., Pop, M., and Salzberg, S. L. (2009). Ultrafast and memory-efficient alignment of short DNA sequences to the human genome. Genome Biol. 10, R25. doi: 10.1186/gb-2009-10-3-r25

Lee, H. C., Chang, S. S., Choudhary, S., Aalto, A. P., Maiti, M., Bamford, D. H., et al. (2009). qiRNA is a new type of small interfering RNA induced by DNA damage. Nature 459, 274-U163. doi: 10.1038/nature08041

Lee, H. C., Li, L., Gu, W., Xue, Z., Crosthwaite, S. K., Pertsemlidis, A., et al. (2010). Diverse pathways generate microRNA-like RNAs and Dicer-independent small interfering RNAs in fungi. Mol. Cell 38, 803-814. doi: 10.1016/ j.molcel.2010.04.005

Lee, A. H., Hurley, B., Felsensteiner, C., Yea, C., Ckurshumova, W., Bartetzko, V., et al. (2012). A bacterial acetyltransferase destroys plant microtubule networks and blocks secretion. PloS Pathog. 8, e1002523. doi: 10.1371/journal.ppat.1002523

Li, F., Pignatta, D., Bendix, C., Brunkard, J. O., Cohn, M. M., Tung, J., et al. (2012). MicroRNA regulation of plant innate immune receptors. Proc. Natl. Acad. Sci. U. S. A. 109, 1790-1795. doi: 10.1073/pnas.1118282109

Liu, J. D., Carmell, M. A., Rivas, F. V., Marsden, C. G., Thomson, J. M., Song, J. J., et al. (2004). Argonaute2 is the catalytic engine of mammalian RNAi. Science 305, 1437-1441. doi: 10.1126/science.1102513

Ma, X., Bologna, N., and Palma-Guerrero, J. (2018a). Small RNA bidirectional crosstalk during the interaction between wheat and Zymoseptoria tritici. bioRxiv 501593. doi: 10.1101/501593

Ma, X., Keller, B., Mcdonald, B. A., Palma-Guerrero, J., and Wicker, T. (2018b). Comparative transcriptomics reveals how wheat responds to infection by Zymoseptoria tritici. Mol. Plant-Microbe Interact. 31 (4), 420-431. doi: 10.1094/MPMI-10-17-0245-R

Marshall, R., Kombrink, A., Motteram, J., Loza-Reyes, E., Lucas, J., HammondKosack, K. E., et al. (2011). Analysis of two in planta expressed LysM effector homologs from the fungus Mycosphaerella graminicola reveals novel functional properties and varying contributions to virulence on wheat. Plant Physiol. 156, 756-769. doi: 10.1104/pp.111.176347

Matzke, M. A., and Mosher, R. A. (2014). RNA-directed DNA methylation: an epigenetic pathway of increasing complexity. Nat. Rev. Genet. 15, 394-408. doi: $10.1038 / \mathrm{nrg} 3683$

Menzies, I. J., Youard, L. W., Lord, J. M., Carpenter, K. L., Van Klink, J. W., Perry, N. B., et al. (2016). Leaf colour polymorphisms: a balance between plant defence and photosynthesis. J. Ecol. 104, 104-113. doi: 10.1111/1365-2745.12494

Mi, S. J., Cai, T., Hu, Y. G., Chen, Y., Hodges, E., Ni, F. R., et al. (2008). Sorting of small RNAs into Arabidopsis argonaute complexes is directed by the 5 c terminal nucleotide. Cell 133, 116-127. doi: 10.1016/j.cell.2008.02.034

Mirzadi Gohari, A., Ware, S. B., Wittenberg, A. H., Mehrabi, R., Ben M’barek, S., Verstappen, E. C., et al. (2015). Effector discovery in the fungal wheat pathogen Zymoseptoria tritici. Mol. Plant Pathol. 16, 931-945. doi: 10.1111/mpp.12251

Moazed, D. (2009). Small RNAs in transcriptional gene silencing and genome defence. Nature 457, 413-420. doi: 10.1038/nature07756

Navarro, L., Jay, F., Nomura, K., He, S. Y., and Voinnet, O. (2008). Suppression of the microRNA pathway by bacterial effector proteins. Science 321, 964-967. doi: 10.1126/science.1159505

Nicolas, F. E., and Ruiz-Vazquez, R. M. (2013). Functional Diversity of RNAi-Associated sRNAs in Fungi. Int. J. Mol. Sci. 14, 15348-15360. doi: 10.3390/ijms140815348

Nolan, T., Braccini, L., Azzalin, G., De Toni, A., Macino, G., and Cogoni, C. (2005). The post-transcriptional gene silencing machinery functions independently of DNA methylation to repress a LINE1-like retrotransposon in Neurospora crassa. Nucleic Acids Res. 33, 1564-1573. doi: 10.1093/nar/gki300

Nowara, D., Gay, A., Lacomme, C., Shaw, J., Ridout, C., Douchkov, D., et al. (2010). HIGS: host-induced gene silencing in the obligate biotrophic fungal pathogen Blumeria graminis. Plant Cell 22, 3130-3141. doi: 10.1105/tpc.110.077040

O'driscoll, A., Kildea, S., Doohan, F., Spink, J., and Mullins, E. (2014). The wheatSeptoria conflict: a new front opening up? Trends In Plant Sci. 19, 602-610. doi: 10.1016/j.tplants.2014.04.011 
Palma-Guerrero, J., Torriani, S. F., Zala, M., Carter, D., Courbot, M., Rudd, J. J., et al. (2016). Comparative transcriptomic analyses of Zymoseptoria tritici strains show complex lifestyle transitions and intraspecific variability in transcription profiles. Mol. Plant Pathol. 17, 845-859. doi: 10.1094/MPMI-07-16-0146-R

Palma-Guerrero, J., Ma, X., Torriani, S. F., Zala, M., Francisco, C. S., Hartmann, F. E., et al. (2017). Comparative +. Mol. Plant Microbe Interact. 30, 231-244. doi: 10.1094/MPMI-07-16-0146-R

Park, G. G., Park, J. J., Yoon, J., Yu, S. N., and An, G. (2010). A RING finger E3 ligase gene, Oryza sativa Delayed Seed Germination 1 (OsDSG1), controls seed germination and stress responses in rice. Plant Mol. Biol. 74, 467-478. doi: 10.1007/s11103-010-9687-3

Pessina, F., and Lowndes, N. F. (2014). The RSF1 histone-remodelling factor facilitates DNA double-strand break by recruiting centromeric and Fanconi Anaemia proteins. PloS Biology. 12 (5), e1001856. doi: 10.1371/ journal.pbio.1001856

Plissonneau, C., Sturchler, A., and Croll, D. (2016). The evolution of orphan regions in genomes of a fungal pathogen of wheat. MBio 7. doi: 10.1128/mBio.01231-16

Plissonneau, C., Hartmann, F. E., and Croll, D. (2018). Pangenome analyses of the wheat pathogen Zymoseptoria tritici reveal the structural basis of a highly plastic eukaryotic genome. BMC Biol. 16, 5. doi: 10.1186/s12915-017-0457-4

Rajagopalan, R., Vaucheret, H., Trejo, J., and Bartel, D. P. (2006). A diverse and evolutionarily fluid set of microRNAs in Arabidopsis thaliana. Genes Dev. 20, 3407-3425. doi: 10.1101/gad.1476406

Raman, V., Simon, S. A., Demirci, F., Nakano, M., Meyers, B. C., and Donofrio, N. M. (2017). Small RNA Functions Are Required for Growth and Development of Magnaporthe oryzae. Mol. Plant Microbe Interact. 30, 517530. doi: 10.1094/MPMI-11-16-0236-R

Robinson, M. D., and Oshlack, A. (2010). A scaling normalization method for differential expression analysis of RNA-seq data. Genome Biol. 11, R25. doi: 10.1186/gb-2010-11-3-r25

Robinson, M. D., Mccarthy, D. J., and Smyth, G. K. (2010). edgeR: a Bioconductor package for differential expression analysis of digital gene expression data. Bioinformatics 26, 139-140. doi: 10.1093/bioinformatics/btp616

Rodrigues, M. L., and Casadevall, A. (2018). A two-way road: novel roles for fungal extracellular vesicles. Mol. Microbiol. 110, 11-15. doi: 10.1111/mmi.14095

Rodrigues, M. L., Nimrichter, L., Oliveira, D. L., Frases, S., Miranda, K., Zaragoza, O., et al. (2007). Vesicular polysaccharide export in Cryptococcus neoformans is a eukaryotic solution to the problem of fungal trans-cell wall transport. Eukaryotic Cell 6, 48-59. doi: 10.1128/Ec.00318-06

Rodrigues, M. L., Nimrichter, L., Oliveira, D. L., Nosanchuk, J. D., and Casadevall, A. (2008). Vesicular trans-cell wall transport in fungi: a mechanism for the delivery of virulence-associated macromolecules? Lipid Insights 2, 27-40. doi: 10.4137/lpi.s1000

Rodrigues, M. L., Godinho, R. M. C., Zamith-Miranda, D., and Nimrichter, L. (2015). Traveling into outer space: unanswered questions about fungal extracellular vesicles. PloS Pathog. 11 (12). doi: 10.1371/journal.ppat.1005240

Romano,N.,andMacino,G.(1992).Quelling:transientinactivationofgeneexpression in Neurospora crassa by transformation with homologous sequences. Mol. Microbiol.6,3343-3353.https://www.ncbi.nlm.nih.gov/pubmed/1484489.

Rudd, J. J., Kanyuka, K., Hassani-Pak, K., Derbyshire, M., Andongabo, A., Devonshire, J., et al. (2015). Transcriptome and metabolite profiling of the infection cycle of Zymoseptoria tritici on wheat reveals a biphasic interaction with plant immunity involving differential pathogen chromosomal contributions and a variation on the hemibiotrophic lifestyle definition. Plant Physiol. 167, 1158-1185. doi: 10.1104/pp.114.255927

Ruiz-Ferrer, V., and Voinnet, O. (2009). Roles of plant small RNAs in biotic stress responses. Annu. Rev. Plant Biol. 60, 485-510. doi: 10.1146/annurev. arplant.043008.092111

Samad, A. F. A., Sajad, M., Nazaruddin, N., Fauzi, I. A., Murad, A. M. A., Zainal, Z., et al. (2017). MicroRNA and transcription factor: key players in plant regulatory network. Front. Plant Sci. 8, 565. doi: 10.3389/fpls.2017.00565

Sanchez-Vallet, A., Mcdonald, M. C., Solomon, P. S., and Mcdonald, B. A. (2015). Is Zymoseptoria tritici a hemibiotroph? Fungal Genet. Biol. 79, 29-32. doi: 10.1016/j.fgb.2015.04.001

Shahid, S., and Axtell, M. J. (2014). Identification and annotation of small RNA genes using ShortStack. Methods 67, 20-27. doi: 10.1016/j.ymeth.2013.10.004

Shivaprasad, P. V., Chen, H. M., Patel, K., Bond, D. M., Santos, B. A., and Baulcombe, D. C. (2012). A microRNA superfamily regulates nucleotide binding site-leucine-rich repeats and other mRNAs. Plant Cell 24, 859-874. doi: 10.1105/tpc.111.095380

Siomi, M. C., Sato, K., Pezic, D., and Aravin, A. A. (2011). PIWI-interacting small RNAs: the vanguard of genome defence. Nat. Rev. Mol. Cell Biol. 12, 246-258. doi: $10.1038 / \mathrm{nrm} 3089$

Sunkar, R., and Zhu, J. K. (2004). Novel and stress-regulated microRNAs and other small RNAs from Arabidopsis. Plant Cell 16, 2001-2019. doi: 10.1105/ tpc. 104.022830

Sunkar, R., Chinnusamy, V., Zhu, J. H., and Zhu, J. K. (2007). Small RNAs as big players in plant abiotic stress responses and nutrient deprivation. Trends In Plant Sci. 12, 301-309. doi: 10.1016/j.tplants.2007.05.001

Torres-Martinez, S., and Ruiz-Vazquez, R. M. (2017). The RNAi universe in fungi: a varied landscape of small RNAs and biological functions. Annu. Rev. Microbiol. Vol7171,371-391. doi: 10.1146/annurev-micro-090816-093352

Tsuda, K., and Somssich, I. E. (2015). Transcriptional networks in plant immunity. New Phytol. 206, 932-947. doi: 10.1111/nph.13286

Vagin, V. V., Sigova, A., Li, C., Seitz, H., Gvozdev, V., and Zamore, P. D. (2006). A distinct small RNA pathway silences selfish genetic elements in the germline. Science 313, 320-324. doi: 10.1126/science.1129333

Vallejo, M. C., Matsuo, A. L., Ganiko, L., Medeiros, L. C., Miranda, K., Silva, L. S., et al. (2011). The pathogenic fungus Paracoccidioides brasiliensis exports extracellular vesicles containing highly immunogenic alpha-Galactosyl epitopes. Eukaryot Cell 10, 343-351. doi: 10.1128/EC.00227-10

Vargas, G., Rocha, J. D. B., Oliveira, D. L., Albuquerque, P. C., Frases, S., Santos, S. S., et al. (2015). Compositional and immunobiological analyses of extracellular vesicles released by Candida albicans. Cell. Microbiol. 17, 389-407. doi: $10.1111 / \mathrm{cmi} .12374$

Wang, M., Weiberg, A., Lin, F. M., Thomma, B. P., Huang, H. D., and Jin, H. (2016). Bidirectional cross-kingdom RNAi and fungal uptake of external RNAs confer plant protection. Nat. Plants 2, 16151. doi: 10.1038/nplants.2016.151

Wang,B.,Sun,Y.,Song,N.,Zhao,M.,Liu,R.,Feng,H.,etal.(2017).Pucciniastriiformisf. sp. tritici microRNA-like RNA 1 (Pst-milR1), an important pathogenicity factor of Pst, impairs wheat resistance to Pst by suppressing the wheat pathogenesisrelated 2 gene. New Phytol. 215, 338-350. doi: 10.1111/nph.14577

Weiberg, A., Wang, M., Lin, F. M., Zhao, H., Zhang, Z., Kaloshian, I., et al. (2013). Fungal small RNAs suppress plant immunity by hijacking host RNA interference pathways. Science 342, 118-123. doi: 10.1126/science.1239705

Weiberg, A., Wang, M., Bellinger, M., and Jin, H. (2014). Small RNAs: a new paradigm in plant-microbe interactions. Annu. Rev. Phytopathol. 52, 495-516. doi: 10.1146/annurev-phyto-102313-045933

Yang, X., and Li, L. (2011). miRDeep-P: a computational tool for analyzing the microRNA transcriptome in plants. Bioinformatics 27, 2614-2615. doi: 10.1093/bioinformatics/btr430

Zeng, W. P., Wang, J., Wang, Y., Lin, J., Fu, Y. P., Xie, J. T., et al. (2018). Dicer-Like proteins regulate sexual development via the Biogenesis of perithecium-specific micrornas in a plant pathogenic Fungus Fusarium graminearum. Front. In Microbiol. 9, 818. doi: 10.3389/fmicb.2018.00818

Zhai, J. X., Jeong, D. H., De Paoli, E., Park, S., Rosen, B. D., Li, Y. P., et al. (2011). MicroRNAs as master regulators of the plant NB-LRR defense gene family via the production of phased, trans-acting siRNAs. Genes Dev. 25, 2540-2553. doi: 10.1101/gad.177527.111

Zhang, W., Gao, S., Zhou, X., Chellappan, P., Chen, Z., Zhou, X., et al. (2011). Bacteriaresponsive microRNAs regulate plant innate immunity by modulating plant hormone networks. Plant Mol. Biol. 75, 93-105. doi: 10.1007/s11103-010-9710-8

Zhang, T., Zhao, Y. L., Zhao, J. H., Wang, S., Jin, Y., Chen, Z. Q., et al. (2016). Cotton plants export microRNAs to inhibit virulence gene expression in a fungal pathogen. Nat. Plants 2, 16153. doi: 10.1038/nplants.2016.153

Conflict of Interest: The authors declare that the research was conducted in the absence of any commercial or financial relationships that could be construed as a potential conflict of interest.

Copyright (c) $2020 \mathrm{Ma}$, Wiedmer and Palma-Guerrero. This is an open-access article distributed under the terms of the Creative Commons Attribution License (CC BY). The use, distribution or reproduction in other forums is permitted, provided the original author(s) and the copyright owner(s) are credited and that the original publication in this journal is cited, in accordance with accepted academic practice. No use, distribution or reproduction is permitted which does not comply with these terms. 